\title{
A genome-wide shRNA screen identifies GAS1 as a novel melanoma metastasis suppressor gene
}

\author{
Stephane Gobeil, ${ }^{1}$ Xiaochun Zhu, ${ }^{1}$ Charles J. Doillon, ${ }^{2}$ and Michael R. Green ${ }^{1,3}$ \\ ${ }^{1}$ Howard Hughes Medical Institute, Programs in Gene Function and Expression and Molecular Medicine, University of \\ Massachusetts Medical School, Worcester, Massachusetts 01605, USA; ${ }^{2}$ Oncology and Molecular Endocrinology Research \\ Center, CHUL's Research Center, CHUQ, Laval University, Quebec City, Québec G1V 4G2, Canada
}

\begin{abstract}
Metastasis suppressor genes inhibit one or more steps required for metastasis without affecting primary tumor formation. Due to the complexity of the metastatic process, the development of experimental approaches for identifying genes involved in metastasis prevention has been challenging. Here we describe a genome-wide RNAi screening strategy to identify candidate metastasis suppressor genes. Following expression in weakly metastatic B16-F0 mouse melanoma cells, shRNAs were selected based upon enhanced satellite colony formation in a three-dimensional cell culture system and confirmed in a mouse experimental metastasis assay. Using this approach we discovered 22 genes whose knockdown increased metastasis without affecting primary tumor growth. We focused on one of these genes, Gas1 (Growth arrest-specific 1), because we found that it was substantially down-regulated in highly metastatic B16-F10 melanoma cells, which contributed to the high metastatic potential of this mouse cell line. We further demonstrated that Gas1 has all the expected properties of a melanoma tumor suppressor including: suppression of metastasis in a spontaneous metastasis assay, promotion of apoptosis following dissemination of cells to secondary sites, and frequent down-regulation in human melanoma metastasis-derived cell lines and metastatic tumor samples. Thus, we developed a genome-wide shRNA screening strategy that enables the discovery of new metastasis suppressor genes.
\end{abstract}

[Keywords: Metastasis suppressor gene; melanoma; GAS1; RNAi screen; three-dimensional cell culture system]

Supplemental material is available at http://www.genesdev.org.

Received July 10, 2008; revised version accepted September 8, 2008.

Metastatic dissemination of a primary tumor to a secondary site is the major cause of deaths from solid tumors (for review, see Gupta and Massague 2006; Nguyen and Massague 2007). The progression to metastasis involves a series of discrete steps, commonly known as the metastatic cascade, which minimally includes: invasion of the tumor border, intravasation into vascular structures, survival during transport to the secondary site, extravasation, and colonization of the secondary site (for review, see Gupta and Massague 2006; Steeg 2006). The complex process of metastasis is controlled by multiple genes that either increase or decrease metastatic potential (Berger et al. 2005; Nguyen and Massague 2007). Although many genes have been identified that promote metastasis, a relatively small number of metastasis suppressor genes have been documented (Rinker-Schaeffer et al. 2006). This is due, at least in part, to a lack of

${ }^{3}$ Corresponding author.

E-MAIL michael.green@umassmed.edu; FAX (508) 856-5473.

Article is online at http://www.genesdev.org/cgi/doi/10.1101/gad.1714608. experimental approaches for the systematic identification of genes that specifically inhibit metastasis.

Three-dimensional (3D) cell culture systems comprising cancer cell lines grown in matrices of collagen and fibrin provide an ex vivo model system for studying tumor cell invasion and expansion into the extracellular matrix (Doillon et al. 2004). Using this bicomposite gel technology system, it has been shown that a variety of "aggressive" human tumor cell lines, including colorectal, prostate, and non-small lung carcinoma cells, rapidly develop satellite colonies separate from the primary tumors. The formation of these satellite colonies provides a cell culture model that recapitulates several critical steps of the metastatic process, including tumor cell motility and invasion, expansion into the collagen matrix, and the ability to survive and form colonies at secondary sites. Here we show how a 3D cell culture system can be used in conjunction with genome-wide RNAi screening to identify candidate metastasis suppressor genes. These candidates can be further analyzed and validated, thereby enabling the discovery of new metastasis suppressor genes in specific cancer types. 


\section{Results}

A genome-wide shRNA screen for the identification of candidate metastasis suppressor genes

Using a 3D cell culture system as an initial selection, we performed a genome-wide shRNA screen to identify genes that, when knocked down, increase formation of satellite colonies (Fig. 1A). A mouse shRNA library comprising $\sim 62,400$ shRNAs directed against $\sim 28,000$ genes (Silva et al. 2005) was divided into 10 pools, which were packaged into retrovirus particles and used to stably transduce poorly metastatic B16-F0 mouse melanoma cells (Fidler 1975). The cells were embedded in collagen, coated with a basement membrane matrix (Matrigel), sandwiched between two layers of fibrin gel to completely encapsulate the collagen/Matrigel plug, and then incubated for $2 \mathrm{wk}$. Figure 1B shows, as expected, that B16-F0 cells produced few (five to 10) satellite colonies whereas B16-F10 cells, a highly metastatic counterpart of B16-F0 cells (Fidler 1975), produced numerous (5001000) satellite colonies. Transduction of B16-F0 cells with an shRNA pool, but not a nonsilencing (NS) shRNA control, also resulted in the appearance of many satellite colonies; the results from a representative pool are shown (Fig. 1C).

Following the 2-wk incubation, satellite colonies from each pool were isolated, genomic DNA was extracted
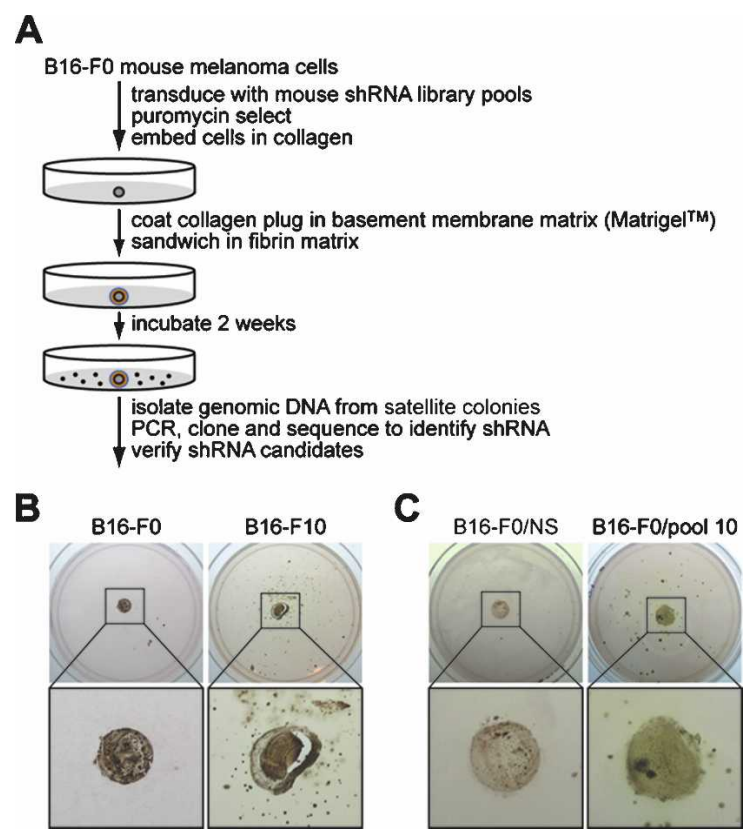

Figure 1. A genome-wide shRNA screen for the identification of candidate metastasis suppressor genes. (A) Schematic summary of the screen. Cells were embedded in collagen (gray), coated in Matrigel (orange), and sandwiched in fibrin (blue). (B) 3D cell culture assay. Collagen/Matrigel-embedded cells are shown in the center, surrounded by migrating satellite colonies in the fibrin matrix. $(C)$ 3D cell culture assay of B16-F0 cells stably transduced with an NS shRNA or a representative shRNA pool. and the shRNAs were identified by sequence analysis. Positive candidates were confirmed in independent single-well 3D cell culture assays by stably transducing B16-F0 cells with a single shRNA directed against each candidate gene (Supplemental Fig. S1; quantified in Supplemental Table S1). In total, the screen identified 78 genes that, following shRNA-mediated knockdown, promoted formation of B16-F0 satellite colonies in the fibrin matrix (Supplemental Table S2).

\section{Identification of 22 genes whose knockdown increase metastasis without affecting primary tumor growth}

We next asked whether knockdown of any of the candidate genes could promote metastasis. B16-F0 cells stably transduced with an shRNA against each of the 78 candidate genes were injected into the tail veins of syngeneic C57BL/6 mice, and $14 \mathrm{~d}$ later the mice were examined for lung metastases. Figure 2A shows, as expected, that injection of B16-F0 cells alone or B16-F0 cells stably expressing an NS shRNA resulted in very few lung metastases (typically $\leq 10$ per lung), whereas injection of B16-F10 cells resulted in a substantially higher number ( 200 per lung). Of the 78 genes identified in the 3D cell culture assay, knockdown of 22 genes led to a significant increase in the number of lung metastases relative to the NS shRNA (Table 1; Fig. 2A, quantitated in B). Quantitative real-time RT-PCR (qRT-PCR) confirmed in all cases that expression of the target gene was decreased in each B16-F0 knockdown cell line (Supplemental Fig. S2A). For three of the genes for which suitable antibodies were available (Gas1 [Growth arrest-specific 1], Acta2, and Ccna2) target gene knockdown was also confirmed by immunoblot analysis (Supplemental Fig. S2B). For all genes, a second, unrelated shRNA directed against the same target also resulted in gene knockdown and increased formation of satellite colonies in the $3 \mathrm{D}$ assay (Supplemental Fig. S3). The 22 genes are involved in a variety of processes including cell cycle regulation (Ccna2 and Gas1), cell communication and signal transduction (Olfr198 and Pdyn), cytoskeletal function (Acta2), development (Dppa3), intracellular transport (Tomm70a), metabolism/energy pathways (Alg6, Hsd3b2, and Phka1), and protein metabolism (Cpa2 and Ctsol.

An essential criterion of a metastasis suppressor gene is that it does not affect growth of the primary tumor (Steeg 2003). We therefore tested whether knocking down any of the candidate genes affected the ability of B16-F0 cells to form primary tumors. B16-F0 cells stably expressing an shRNA directed against each candidate gene were subcutaneously injected into the flanks of C57BL/6 mice, and tumor volume was measured at day 16. Figure $2 \mathrm{C}$ shows that knockdown of any of the 22 genes did not significantly affect primary tumor growth.

Down-regulation of Gas1 in B16-F10 cells contributes to their high metastatic potential

Metastasis suppressor genes are often expressed at lower levels in highly metastatic tumor cells relative to poorly 
Gobeil et al.

A

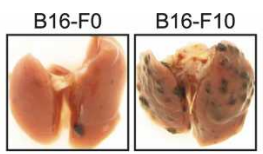

B16-F0

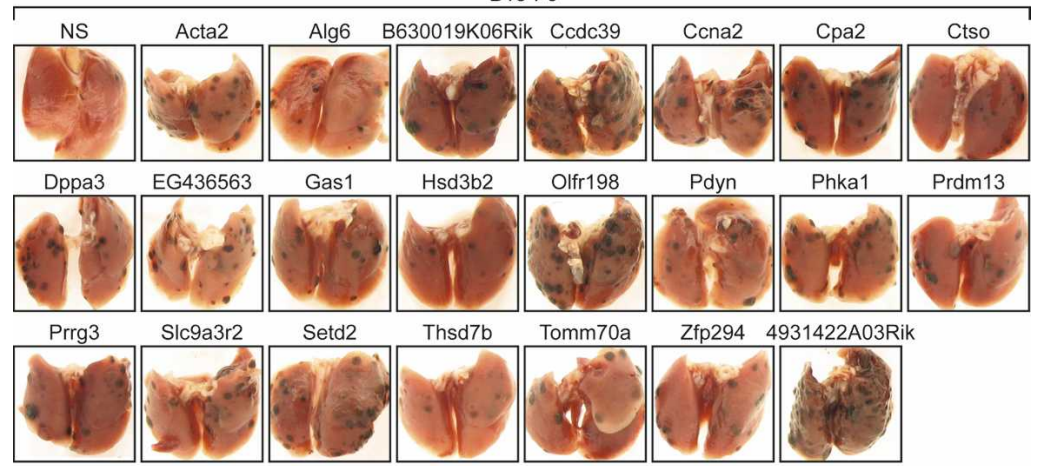

B

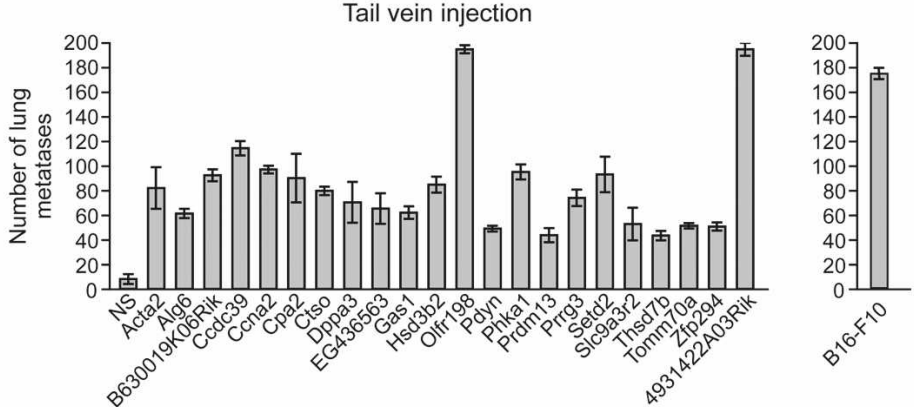

Figure 2. Identification of 22 genes whose knockdown increase metastasis without affecting primary tumor growth. (A) Mouse tail vein metastasis assays showing lungs from mice injected with B16-F0 or B16-F10 cells, or B16-F0 cells stably expressing an NS shRNA or an shRNA directed against one of 22 genes. $(B)$ Quantitation of the results presented in $A$. Error bars represent SEM ( $n=3$ mice). $(C)$ Subcutaneous flank injections. Primary tumor growth at day 16 following subcutaneous injection of B16-F0 KD cell lines. Error bars represent SEM $(n=3$ mice).

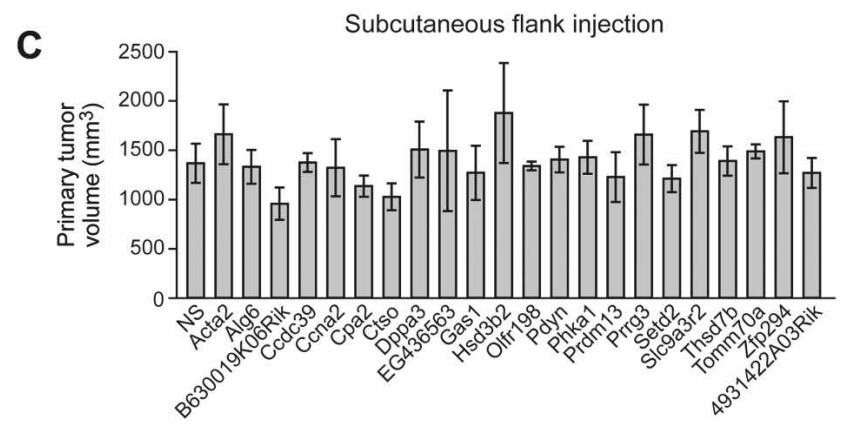

metastatic or nonmetastatic tumor cells or normal cells (for review, see Steeg 2003). To test whether any of the 22 candidate genes conformed to this pattern, we first compared their expression in highly metastatic B16-F10 and poorly metastatic B16-F0 cells. The qRT-PCR results of Figure $3 \mathrm{~A}$ show that all but one of the genes were either expressed equally or at most modestly down-regulated in B16-F10 cells compared with B16-F0 cells. By contrast, one gene, Gas1, was markedly down-regulated ( 11-fold) in B16-F10 cells. Gas1 encodes a pleiotropic 45-kDa glycosylphosphatidylinositol-anchored membrane protein (Stebel et al. 2000) that has been shown to play a role in both negative (Del Sal et al. 1992; Lee et al. 2001; Mellstrom et al. 2002; Zamorano et al. 2003, 2004) and positive regulation of cell growth (Liu et al. 2001; Spagnuolo et al. 2004), as well as in embryonic development through regulation of Sonic Hedgehog signaling (for review, see Martinelli and Fan 2007b).
To determine whether down-regulation of Gas1 contributed to the high metastatic capacity of B16-F10 cells, we tested whether ectopic expression of Gas1 in B16-F10 cells could reduce their ability to metastasize. Significantly, ectopic expression of Gas1 decreased the number of satellite colonies in the $3 \mathrm{D}$ cell culture assay (Fig. 3B) and the number of lung metastases in the mouse tail vein metastasis assay (Fig. $3 \mathrm{C}$ ) relative to the empty vector control. Ectopic Gas1 expression had no effect on the ability of B16-F10 cells to form a tumor when injected subcutaneously in mice (Fig. 3D), indicating that the reduced metastatic efficiency following ectopic expression of Gas1 in B16-F10 cells was not the result of a general decrease in cell proliferation or tumorigenicity. Consistent with this finding, cultured B16-F0 cells expressing an NS shRNA (B16-F0/NS) and B16-F0 Gas1 knockdown (B16-F0/Gas1 KD) cells have comparable proliferation rates and levels of apoptosis (Supplemental Fig. S4). 
Table 1. Candidate metastasis suppressor genes that tested positive in the mouse tail vein metastasis assay

\begin{tabular}{lll}
\hline Biological function & Gene symbol & \\
\hline Cell cycle regulation & Ccna2 & Cyclin A2 \\
& Gas1 & Growth arrest-specific 1 \\
Cell communication/signal & Olfr198 & Olfactory receptor 198 \\
transduction & Pdyn & Prodynorphin \\
Cytoskeleton & Acta2 & Actin, $\alpha$ 2, smooth muscle, aorta \\
Development & Dppa3 & Developmental pluripotency-associated 3 \\
Transport & Tomm70a & Translocase of outer mitochondrial membrane 70 homolog A (yeast) \\
Metabolism/energy pathways & Alg6 & Asparagine-linked glycosylation 6 homolog (yeast) \\
& Hsd3b2 & Hydroxy- $\delta$-5-steroid dehydrogenase, 3 $\beta$ - and steroid $\delta$-isomerase 2 \\
& Phka1 & Phosphorylase kinase $\alpha$ 1 \\
Protein metabolism & Cpa2 & Carboxypeptidase A2, pancreatic \\
Unknown & Ctso & Cathepsin O \\
& $4931422 A 03 R i k$ & RIKEN cDNA 4931422A03 gene \\
& B630019K06Rik & RIKEN cDNA B630019K06 gene \\
& Ccdc39 & Coiled-coil domain containing 39 \\
& EG436563 & Predicted gene, EG436563 \\
& Prdm13 & PR domain containing 13 \\
& Prrg3 & Proline-rich G-carboxyglutamic acid protein 3 \\
& Setd2 & SET domain containing 2 \\
& Slc9a3r2 & Solute carrier family 9 (sodium/hydrogen exchanger), \\
& Thsd7b & isoform 3 regulator 2 \\
& Zfp294 & Thrombospondin, type I, domain containing 7B \\
& & Zinc finger protein 294 \\
\end{tabular}

Gas1 knockdown promotes metastasis in a spontaneous metastasis assay

The tail vein injection assay bypasses several critical steps of the metastatic cascade such as invasion of the tumor border and intravasation into the vasculature. We therefore tested whether Gas1 knockdown could promote metastasis in a more rigorous "spontaneous metastasis assay" in which B16-F0 cells, stably transduced with a Gas1 or NS shRNA, were injected subcutaneously into the foot pad of C57BL/6 mice and approximately 6 wk later the animals were examined for lung metastases.
Figure 4 shows that the percentage of animals developing lung metastases was substantially higher in mice injected with B16-F0/Gas1 KD cells than those injected with B16-F0/NS cells.

Gas1 suppresses metastasis by promoting apoptosis of cells following dissemination to secondary sites

One way in which a metastasis suppressor can function is by preventing tumor cells from colonizing or surviving at secondary sites. To test whether Gas1 functioned in

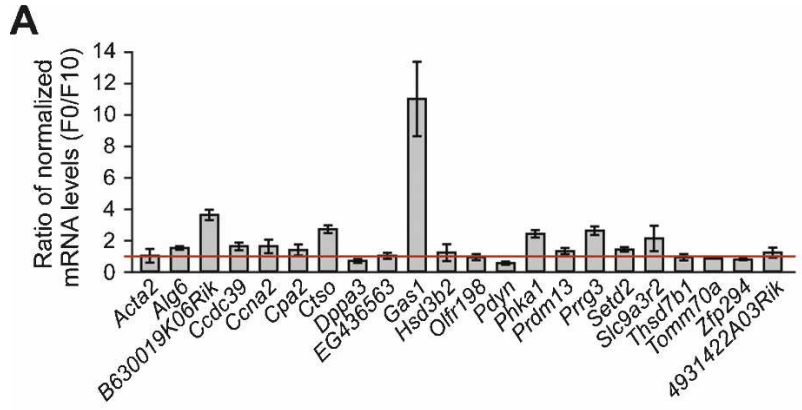

B

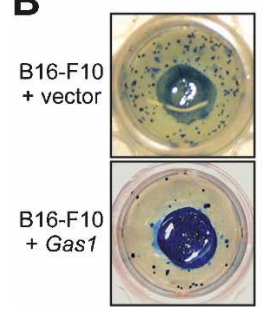

C

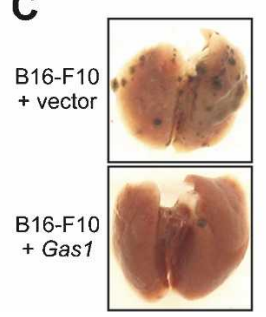

D

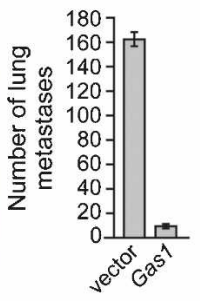

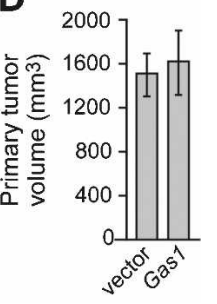

Figure 3. Down-regulation of Gas1 in B16-F10 cells contributes to their high metastatic potential. $(A) \mathrm{qRT}-$ PCR analysis of candidate gene expression in B16-F0 and B16-F10 cells. Gene expression was normalized to Gapdh, and presented as the ratio of expression in B16$\mathrm{F} 0$ relative to $\mathrm{B} 16-\mathrm{F} 10$ cells. A ratio $>1$ labove the red line) indicates down-regulation in B16-F10 cells. Error bars represent $\mathrm{SD}(n=3)$. (B) $3 \mathrm{D}$ cell culture assay showing B16-F10 cells stably expressing Gas1 or vector. $(C$, left $)$ Mouse tail vein metastasis assay showing lungs from mice injected with B16-F10 cells stably expressing Gas1 or vector. (Right) Quantitation. Error bars represent SEM ( $n=3$ mice). (D) Primary tumor growth at day 16 following subcutaneous injection of B16-F10 cells stably expressing Gas1 or vector. Error bars represent $\operatorname{SEM}(n=3$ mice $)$. 
Gobeil et al.

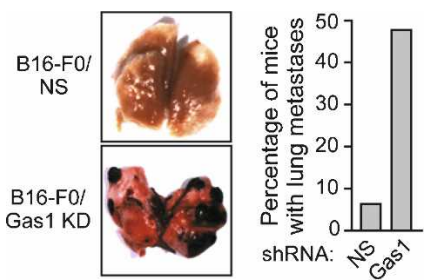

Figure 4. Gas1 knockdown promotes metastasis in a spontaneous metastasis assay. (Left) Spontaneous metastasis assay showing lungs from mice injected with B16-F0 cells stably expressing a Gas1 or NS shRNA. (Right) Percentage of mice injected with B16-F0/NS $(n=16)$ or B16-F0/Gas1 KD $(n=21)$ cells with lung metastases.

such a manner, we labeled B16-F0/NS and B16-F0/Gas1 $\mathrm{KD}$ cells with a fluorescent dye and quantified their persistence after arrival in the lung following tail vein injection. Figure $5 \mathrm{~A}$ shows that $1 \mathrm{~h}$ after injection, the number of B16-F0/Gas1 KD cells and B16-F0/NS cells in the lung was roughly equivalent, whereas at later times, three (Fig. 5A) or 24 (Supplemental Fig. S5) hours after injection, the number of B16-F0/Gas1 KD cells that remained in the lung was significantly higher than the number of B16-FO/NS cells. Notably, apoptosis was reduced in B16-F0/Gas1 KD cells compared with B16-F0/ NS cells, as evidenced by decreased levels of activated caspase (Fig. 5B). Collectively, these results suggest that Gas1 suppresses metastasis by promoting apoptosis of cells following dissemination to secondary sites.

In support of this conclusion, ectopic expression of the anti-apoptotic protein Bcl-2 in B16-F0 cells resulted in an increased number of satellite colonies in the $3 \mathrm{D}$ assay (Supplemental Fig. S6), similar to the effect observed upon Gas1 knockdown. Notably, previous studies have shown that Bcl-2 expression in B16 melanoma cells blocks apoptosis and increases the number of pulmonary metastases following mouse tail vein injection (Takaoka et al. 1997).

\section{GAS1 is down-regulated in human metastatic melanoma cell lines and tissue samples}

Acquisition of metastatic potential is thought to involve the inactivation or down-regulation of metastasis suppressor genes (Berger et al. 2005; Nguyen and Massague 2007). We therefore asked whether progression of human primary to metastatic melanoma might involve loss of GAS1. In support of this idea, a search of the publicly accessible Oncomine cancer profiling database (Rhodes et al. 2007) revealed that GAS1 was significantly downregulated $(P \leq 0.05)$ in metastatic melanoma compared with primary melanoma and benign nevi (Fig. 6A; Haqq et al. 2005).

To confirm and extend this observation, we monitored GAS1 expression in a panel of human melanoma cell lines that originated from either a primary tumor or a metastasis. The immunoblot of Figure 6B shows that GAS1 expression was high in UACC-257 cells, derived from a primary melanoma, and markedly reduced in melanoma cell lines derived from metastases isolated from lymph nodes (LOX IMVI and SK-MEL-5), lung (MALME-3M), or skin (SK-MEL-2). Significantly, knockdown of GAS1 in UACC-257 cells increased their ability to form satellite colonies in the 3D cell culture assay (Supplemental Fig. S7), analogous to the results with B16-F0 cells. Finally, we also monitored expression of GAS1 in a pair of well-characterized, experimentally derived human melanoma cell lines: Mel-STV and MelSTR. Mel-STV cells are immortalized primary human melanocytes that can be transformed by expression of an oncogenic RasV12 allele and the resulting RasV12-transformed cells (Mel-STR cells) can form metastatic tumors in vivo (Gupta et al. 2005). We found that GAS1 expression was reduced approximately sixfold in Mel-STR cells relative to Mel-STV cells (Supplemental Fig. S8).

Next, we analyzed GAS1 expression in human primary and metastatic melanoma samples using a melanoma tissue microarray. As a control, samples were stained with the melanoma marker HMB45 (Kapur et al. 1992). Figure 6C shows that GAS1 expression was detectable in the majority $(-83 \%)$ of primary melanomas but in only $\sim 41 \%$ of metastatic melanomas. Collectively, these results indicate that GAS1 expression is frequently down-regulated during progression to metastatic melanoma.

\section{Discussion}

In this study, we described an experimental strategy for the systematic identification of candidate metastasis

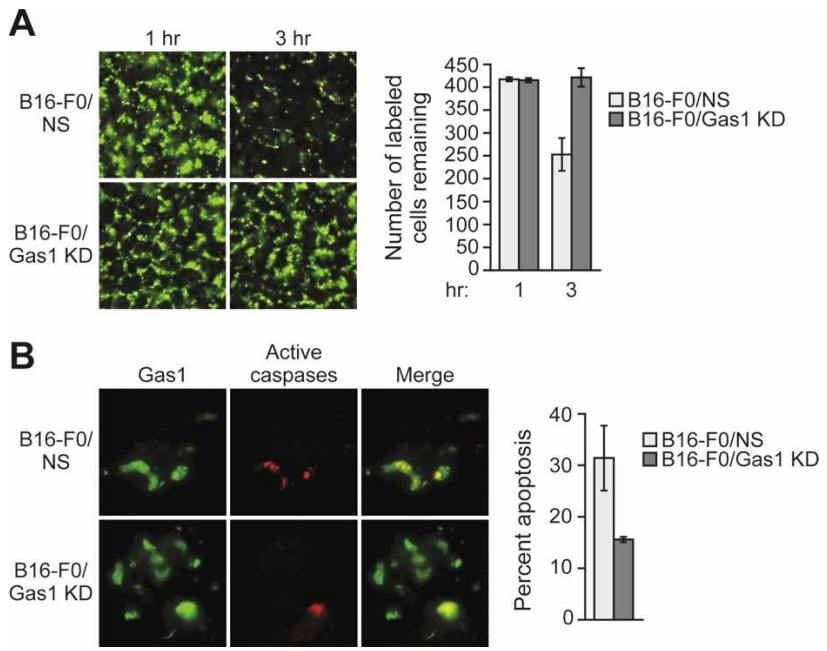

Figure 5. Gas1 suppresses metastasis by promoting apoptosis of cells following dissemination to secondary sites. $(A$, left $)$ In vivo single-cell fluorescence imaging of the lung following tail vein injection of fluorescently labeled B16-F0 cells stably expressing a Gas1 or NS shRNA. (Right) Quantitation; error bars represent SEM ( $n=3$ mice); at $1 \mathrm{~h} P=0.7364$ and at $3 \mathrm{~h}$ $P=0.0148$. $(B$, left $)$ Representative images of B16-F0/NS or B16F0/Gas1 KD cells colocalizing with a fluorescent pan-caspase probe, monitored at $3 \mathrm{~h}$ after injection. (Right) Quantitation of the percentage of apoptotic B16-F0/NS or B16-F0/Gas1 KD cells. Error bars represent SEM $(n=3$ mice). 
A

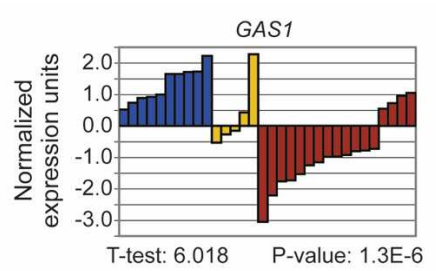

B

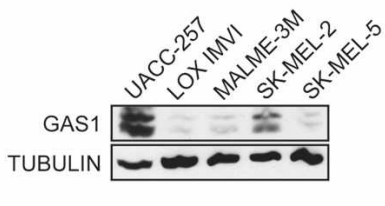

C
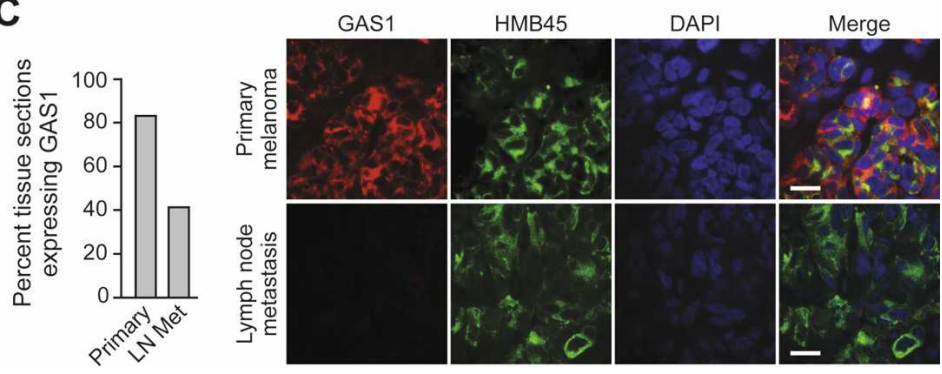

Figure 6. Down-regulation of GAS1 in human metastatic melanoma cell lines and tissue samples. $(A)$ GAS1 expression in benign nevi (blue), primary melanoma (yellow), and metastatic melanoma (red). Data were obtained through the Oncomine database; a Student's $t$-test, performed directly though the Oncomine 3.0 software, showed the difference in gene expression between the samples was significant. $(B)$ Immunoblot analysis of GAS1 expression in human melanoma cell lines derived from primary tumors (UACC-257) or metastatic sites (LOX IMVI, MALME-3M, SK-MEL-2, and SK-MEL-5). Tubulin was monitored as a loading control. $(C$, left $)$ Quantitation of detectable GAS1 expression in primary $(n=47)$ and lymph node metastatic $(n=17)$ melanoma samples. (Right) Representative examples of a primary melanoma and lymph node metastasis analyzed for expression of GAS1 (red) and the melanoma marker HMB45 (green); nuclei were stained with DAPI (blue). Merged images are shown. Bar, $20 \mu \mathrm{m}$. suppressor genes. Using this approach, we found 78 genes that inhibited formation of satellite colonies in a $3 \mathrm{D}$ cell culture assay and showed that 22 of these genes suppressed metastasis to the lung following mouse tail vein injection. The other 56 genes we identified could function as metastasis suppressors for colonization of organs other than the lung or in alternative steps of the metastatic cascade that were not tested here.

Of these 22 new metastasis suppressor gene candidates we focused on Gas1 because we found that it was downregulated in highly metastatic B16-F10 melanoma cells and that this down-regulation contributed to the high metastatic potential of this mouse cell line. We further demonstrated that Gas1 has the expected properties of a melanoma tumor suppressor including suppression of metastasis in both experimental (Fig. 2) and spontaneous assays (Fig. 4), promotion of apoptosis following dissemination of cells to secondary sites (Fig. 5B), and frequent down-regulation in human metastasis-derived cell lines and metastatic tumor samples (Fig. 6A-C). The basis by which GAS1 is down-regulated in metastatic melanoma remains to be determined. Preliminary analysis of the GAS1 promoter reveals a CpG island (S. Gobeil and M.R. Green, unpubl.), raising the possibility that downregulation of GAS1 expression is due to epigenetic silencing.

Our results indicate that Gas1 suppresses metastasis, at least in part, by promoting apoptosis following dissemination of cells to secondary sites. To date, we have found no evidence for a role of Gas 1 in other steps in the metastatic cascade, such as invasion (Supplemental Fig. S9|. Consistent with our results, previous studies have reported that Gas1 can have a proapoptotic activity in specific cell types (Mellstrom et al. 2002; Zamorano et al. 2004). In addition, we note that other metastasis suppressors such as Caspase 8, BRMS1, and TIMPs have, like Gas1, been shown to prevent apoptosis following dissemination to secondary sites (for review, see Stafford et al. 2008). The detailed mechanism by which Gas1 promotes apoptosis at the secondary site remains to be de- termined and there are several reasonable possibilities. For example, loss of Gas1 could facilitate immune evasion, increase survival from biophysical forces encountered during metastatic spread, or regulate the response to cell death or survival signals emanating from the microenvironment at the secondary site.

The signaling pathways by which Gas1 induces apoptosis remain unknown. Previous studies have shown that during development Gas1 binds the secreted morphogen Sonic Hedgehog (Shh) with high affinity (Lee et al. 2001) and regulates Shh signaling (Lee et al. 2001; Cobourne et al. 2004; Allen et al. 2007; Martinelli and Fan 2007a; Seppala et al. 2007). Aberrant activation of Shh signaling has been implicated in the development of several cancers (for review, see Wetmore 2003) including melanoma (Stecca et al. 2007). However, we found that Shh signaling was not affected by either Gas1 knockdown in B16-F0 cells or ectopic expression of Gas1 in B16-F10 cells as evidenced by levels of the well-characterized Shh marker Gli1 (Supplemental Fig. S10). Interestingly, Gas1 has been reported to bind the receptor tyrosine kinase Ret and to inhibit Ret-mediated survival signaling pathways in neuroblastoma cells (Cabrera et al. 2006; Lopez-Ramirez et al. 2008). Whether Gas1 induces melanoma cell apoptosis through a similar mechanism remains to be determined.

Although we have not yet further pursued the other 21 genes, a search of the publicly accessible Oncomine cancer profiling database revealed that in addition to GAS1, three genes (ACTA2, CTSO, and SLC9A3R2) were also significantly down-regulated $(P \leq 0.05)$ in metastatic melanoma compared with primary melanoma and/or benign nevi (Supplemental Fig. S11A; Haqq et al. 2005). Moreover, DPPA3 is expressed at significantly lower levels in advanced relative to early stage melanoma (Supplemental Fig. S11B; Smith et al. 2005). A search of other cancer types revealed that 15 of the genes we identified, including GAS1, were down-regulated in either metastatic versus primary tumor samples, or in late (stages III and IV) relative to early (stage I) disease in multiple can- 
cer types (Supplemental Table S3). Thus, it seems highly likely that some of these other 21 genes are also metastasis suppressors in melanomas or other cancers.

Our search of other cancer types showed that, in addition to melanoma, GAS1 is also down-regulated in breast and prostate cancer metastases (Supplemental Table S3). Consistent with this observation, we found that knockdown of Gas1 increased the ability of weakly metastatic mouse breast cancer 67NR cells (Aslakson and Miller 1992 ) to colonize the lung following mouse tail vein injection (Supplemental Fig. S12), analogous to the results with B16-F0 melanoma cells. In addition, down-regulation of GAS1 is associated with the progression of prostate cancer (Bettuzzi et al. 2000; Scaltriti et al. 2004). These considerations strongly suggest that GAS1 is also a metastasis suppressor gene in cancers other than melanoma.

\section{Materials and methods}

Cell lines and cell culture

Mouse melanoma cell lines B16-F0 (American Type Culture Collection [ATCC] \#CRL-6322) and B16-F10 (ATCC \#CRL6475) were maintained in Dulbecco's modified Eagle's medium supplemented with $10 \%$ fetal calf serum at $37^{\circ} \mathrm{C}$ and $5 \% \mathrm{CO}_{2}$. To generate stable Gas1-expressing B16-F10 cell lines, Gas1 was subcloned from the expression vector pcDNA3-Gas1 (a gift from C.M. Fan, Carnegie Institution of Washington) into the retroviral vector pQCXI-puro (Clontech). Melanoma cell lines UACC257, LOX IMVI, SK-MEL-2, SK-MEL-5, and MALME-3M were obtained from ATCC and grown as recommended by the supplier.

\section{$3 D$ cell culture assay}

Cells were embedded in collagen gel at a density of $5 \times 10^{4}$ cells per $200 \mu \mathrm{L}$ of gel in 96-well plates, as described previously (Doillon et al. 2004). After 2 to $3 \mathrm{~h}$, the gels were removed from the well, soaked in growth factor-reduced Matrigel (BD Biosciences) for $2 \mathrm{~min}$, sandwiched into a fibrin gel laid down in the wells of a 24-well (or 10-cm) plate, and incubated for $14 \mathrm{~d}$ at $37^{\circ} \mathrm{C}$ with $5 \% \mathrm{CO}_{2}$ in culture medium. The media was renewed every other day, and the ability of cells to migrate into the fibrin was assessed every other day. The anti-fibrinolytic agent aprotinin (Sigma) was added to the culture media at $100 \mathrm{U} / \mathrm{mL}$. Satellite colonies were stained using a solution of $0.2 \%$ methylene blue in $50 \%$ methanol.

To perform the shRNA screen, retroviral pools were prepared as described previously (Gazin et al. 2007). Satellite colonies were isolated from the fibrin matrix and expanded for genomic DNA isolation. A minimum of 96 clones were sequenced per pool, as described (Gazin et al. 2007). Using this approach we initially identified 113 candidate genes, which were retested by $\mathrm{g}$ previouslyenerating individual knockdown cell lines by retroviral transduction of $2 \times 10^{5}$ B16-F0 cells (in six-well plates) with the respective shRNA. shRNAs were either obtained from the Open Biosystems library or the RNAi Consortium (Supplemental Table S4). A total of 78 candidates reconfirmed in the independent, single-well 3D cell culture assays.

\section{Metastasis assays}

For the experimental assay, $2 \times 10^{5}$ cells were suspended in 200 $\mu \mathrm{L}$ of PBS and injected in the lateral tail vein of three C57BL/6 mice (Taconic). Lungs were harvested $14 \mathrm{~d}$ post-injection and fixed in formalin. Metastases were counted and statistical analysis (one-way ANOVA) was performed using GraphPad Prism version 3.00 for Windows (GraphPad Software). Experiments were performed in accordance with the Institutional Animal Care and Use Committee (IACUC) guidelines. For the spontaneous metastasis assay, $1 \times 10^{6}$ cells were injected into the footpad of syngeneic C57BL/6 mice. When the primary tumor reached a size of $100 \mathrm{~mm}^{3}$ it was excised, and the mice were examined for lung metastases 4 wk later. Experiments were performed in accordance with the guidelines of the Canadian Council for Animal Care and approved by Laval University Institutional Animal Care Committee.

\section{Tumor formation assays}

Tumor formation assays were performed essentially as described previously (Gazin et al. 2007). Briefly, $5 \times 10^{4}$ cells were suspended in $60 \mu \mathrm{L}$ Matrigel and injected subcutaneously into the right flank of C57BL/6 mice (three mice per shRNA). Tumor volume was calculated as described (Gazin et al. 2007). Statistical analysis was performed as described above.

\section{$q R T-P C R$}

To monitor target gene knockdown, total RNA was isolated $7 \mathrm{~d}$ following retroviral transduction and puromycin selection, and qRT-PCR was performed as described previously (Gazin et al. 2007). Primer sequences used for qRT-PCR are provided in Supplemental Table S5.

\section{Single-cell fluorescence imaging}

Experiments were performed essentially as previously described (Tsai et al. 2007). B16-F0/NS or B16-F0/Gas1 KD cells $\left(1 \times 10^{6}\right)$ were fluorescently labeled with CellTracker Green (Invitrogen) and injected into the tail vein of C57BL/6 mice. At various time points after injection, mice were euthanized and their lungs fixed in formalin followed by imaging using a Zeiss Axiophot 2 fluorescence microscope. Using ImageJ software, the number of events $>10$ pixels were counted in five random fields per lung ( $n=3$ mice), and statistical analysis (Student's $t$-test) was performed using GraphPad Prism version 3.00 for Windows (GraphPad Software). To monitor caspase activation, at $3 \mathrm{~h}$ after injection of B16-F0/NS or B16-F0/Gas1 KD cells mice were injected with a sulforhodamine-conjugated fluoromethylketone derivative of Val-Ala-Asp (Immunochemistry) and sacrificed $30 \mathrm{~min}$ later. The lungs were excised, washed in PBS, and snap frozen in OCT embedding medium. Cryosections $(10 \mu \mathrm{m})$ were analyzed by fluorescence microscopy. Only cells that retained CellTracker Green were counted $(\sim 100$ per lung; $n=3$ mice $)$. Cells showing both green and red fluorescence were scored as apoptotic.

\section{Tissue microarray analysis}

Immunofluorescence was performed using the ME1001 malignant melanoma, metastatic malignant melanoma, and nevus tissue array (Biomax), which contains samples from 56 cases of malignant melanoma, 20 cases of metastatic (lymph node or fatty tissue) malignant melanoma, and 24 cases of nevus (normal tissue). The array was hybridized overnight at $4^{\circ} \mathrm{C}$ with a biotinylated anti-human GAS1 affinity-purified polyclonal antibody (RD System, \#BAF2636) followed by incubation with a Cy3-conjugated secondary antibody (Sigma, ExtrAvidin-Cy3 \#E4142) for $1 \mathrm{~h}$ at room temperature. As a control, the micro- 
array was incubated for $1 \mathrm{~h}$ at room temperature with a mouse anti-human HMB45 monoclonal antibody (Dako \#M0634), followed by incubation with a FITC-conjugated secondary antibody (Invitrogen, Alexa Fluor 488 goat anti-mouse \#A21121) for $1 \mathrm{~h}$ at room temperature. Cell nuclei were visualized with 4',6diamidino-2-phenylindole (DAPI). Imaging was done by fluorescence microscopy as described above. Only HMB45-positive samples were scored for GAS1 expression.

\section{Oncomine database searches}

The Haqq (Haqq et al. 2005) melanoma microarray data set was accessed using the Oncomine Cancer Profiling Database (http:// www.oncomine.org), and includes 10 normal mole, five primary melanoma, and 17 metastatic melanoma samples. Histograms depicting GAS1 gene expression in each sample, as well as a Student's $t$-test giving a $P$ value for the comparison of candidate gene expression between the groups, were obtained directly through the Oncomine 3.0 software.

\section{Acknowledgments}

We thank F. Miller, C.M. Fan, G. Nolan, R.A. Weinberg, and S. Cory for reagents; S. Griggs for excellent technical assistance; and S. Evans for editorial assistance. M.R.G. is an investigator of the Howard Hughes Medical Institute.

\section{References}

Allen, B.L., Tenzen, T., and McMahon, A.P. 2007. The Hedgehog-binding proteins Gas1 and Cdo cooperate to positively regulate Shh signaling during mouse development. Genes \& Dev. 21: 1244-1257.

Aslakson, C.J. and Miller, F.R. 1992. Selective events in the metastatic process defined by analysis of the sequential dissemination of subpopulations of a mouse mammary tumor. Cancer Res. 52: 1399-1405.

Berger, J.C., Vander Griend, D.J., Robinson, V.L., Hickson, J.A., and Rinker-Schaeffer, C.W. 2005. Metastasis suppressor genes: From gene identification to protein function and regulation. Cancer Biol. Ther. 4: 805-812.

Bettuzzi, S., Davalli, P., Astancolle, S., Carani, C., Madeo, B., Tampieri, A., and Corti, A. 2000. Tumor progression is accompanied by significant changes in the levels of expression of polyamine metabolism regulatory genes and clusterin (sulfated glycoprotein 2) in human prostate cancer specimens. Cancer Res. 60: 28-34.

Cabrera, J.R., Sanchez-Pulido, L., Rojas, A.M., Valencia, A., Manes, S., Naranjo, J.R., and Mellstrom, B. 2006. Gas1 is related to the glial cell-derived neurotrophic factor family receptors $\alpha$ and regulates Ret signaling. J. Biol. Chem. 281: 14330-14339.

Cobourne, M.T., Miletich, I., and Sharpe, P.T. 2004. Restriction of sonic hedgehog signalling during early tooth development. Development 131: 2875-2885.

Del Sal, G., Ruaro, M.E., Philipson, L., and Schneider, C. 1992. The growth arrest-specific gene, gas 1 , is involved in growth suppression. Cell 70: 595-607.

Doillon, C.J., Gagnon, E., Paradis, R., and Koutsilieris, M. 2004. Three-dimensional culture system as a model for studying cancer cell invasion capacity and anticancer drug sensitivity. Anticancer Res. 24: 2169-2177.

Fidler, I.J. 1975. Biological behavior of malignant melanoma cells correlated to their survival in vivo. Cancer Res. 35: $218-224$.
Gazin, C., Wajapeyee, N., Gobeil, S., Virbasius, C.M., and Green, M.R. 2007. An elaborate pathway required for Rasmediated epigenetic silencing. Nature 449: 1073-1077.

Gupta, G.P. and Massague, J. 2006. Cancer metastasis: Building a framework. Cell 127: 679-695.

Gupta, P.B., Kuperwasser, C., Brunet, J.P., Ramaswamy, S., Kuo, W.L., Gray, J.W., Naber, S.P., and Weinberg, R.A. 2005. The melanocyte differentiation program predisposes to metastasis after neoplastic transformation. Nat. Genet. 37: 10471054.

Haqq, C., Nosrati, M., Sudilovsky, D., Crothers, J., Khodabakhsh, D., Pulliam, B.L., Federman, S., Miller 3rd, J.R., Allen, R.E., Singer, M.I., et al. 2005. The gene expression signatures of melanoma progression. Proc. Natl. Acad. Sci. 102: 6092-6097.

Kapur, R.P., Bigler, S.A., Skelly, M., and Gown, A.M. 1992. Anti-melanoma monoclonal antibody HMB45 identifies an oncofetal glycoconjugate associated with immature melanosomes. J. Histochem. Cytochem. 40: 207-212.

Lee, C.S., Buttitta, L., and Fan, C.M. 2001. Evidence that the WNT-inducible growth arrest-specific gene 1 encodes an antagonist of sonic hedgehog signaling in the somite. Proc. Nat1. Acad. Sci. 98: 11347-11352.

Liu, Y., May, N.R., and Fan, C.M. 2001. Growth arrest specific gene 1 is a positive growth regulator for the cerebellum. Dev. Biol. 236: 30-45.

Lopez-Ramirez, M.A., Dominguez-Monzon, G., Vergara, P., and Segovia, J. 2008. Gas1 reduces Ret tyrosine 1062 phosphorylation and alters GDNF-mediated intracellular signaling. Int. J. Dev. Neurosci. 26: 497-503.

Martinelli, D.C. and Fan, C.M. 2007a. Gas1 extends the range of Hedgehog action by facilitating its signaling. Genes \& Dev. 21: 1231-1243.

Martinelli, D.C. and Fan, C.M. 2007b. The role of Gas1 in embryonic development and its implications for human disease. Cell Cycle 6: 2650-2655.

Mellstrom, B., Cena, V., Lamas, M., Perales, C., Gonzalez, C., and Naranjo, J.R. 2002. Gas1 is induced during and participates in excitotoxic neuronal death. Mol. Cell. Neurosci. 19: $417-429$

Nguyen, D.X. and Massague, J. 2007. Genetic determinants of cancer metastasis. Nat. Rev. Genet. 8: 341-352.

Rhodes, D.R., Kalyana-Sundaram, S., Mahavisno, V., Varambally, R., Yu, J., Briggs, B.B., Barrette, T.R., Anstet, M.J., Kincead-Beal, C., Kulkarni, P., et al. 2007. Oncomine 3.0: Genes, pathways, and networks in a collection of 18,000 cancer gene expression profiles. Neoplasia 9: 166-180.

Rinker-Schaeffer, C.W., O'Keefe, J.P., Welch, D.R., and Theodorescu, D. 2006. Metastasis suppressor proteins: Discovery, molecular mechanisms, and clinical application. Clin. Cancer Res. 12: 3882-3889.

Scaltriti, M., Brausi, M., Amorosi, A., Caporali, A., D'Arca, D., Astancolle, S., Corti, A., and Bettuzzi, S. 2004. Clusterin (SGP-2, ApoJ) expression is downregulated in low- and highgrade human prostate cancer. Int. J. Cancer 108: 23-30.

Seppala, M., Depew, M.J., Martinelli, D.C., Fan, C.M., Sharpe, P.T., and Cobourne, M.T. 2007. Gas1 is a modifier for holoprosencephaly and genetically interacts with sonic hedgehog. J. Clin. Invest. 117: 1575-1584.

Silva, J.M., Li, M.Z., Chang, K., Ge, W., Golding, M.C., Rickles, R.J., Siolas, D., Hu, G., Paddison, P.J., Schlabach, M.R., et al. 2005. Second-generation shRNA libraries covering the mouse and human genomes. Nat. Genet. 37: 1281-1288.

Smith, A.P., Hoek, K., and Becker, D. 2005. Whole-genome expression profiling of the melanoma progression pathway reveals marked molecular differences between nevi/mela- 
Gobeil et al.

noma in situ and advanced-stage melanomas. Cancer Biol. Ther. 4: 1018-1029.

Spagnuolo, R., Corada, M., Orsenigo, F., Zanetta, L., Deuschle, U., Sandy, P., Schneider, C., Drake, C.J., Breviario, F., and Dejana, E. 2004. Gas1 is induced by VE-cadherin and vascular endothelial growth factor and inhibits endothelial cell apoptosis. Blood 103: 3005-3012.

Stafford, L.J., Vaidya, K.S., and Welch, D.R. 2008. Metastasis suppressors genes in cancer. Int. J. Biochem. Cell Biol. 40: 874-891.

Stebel, M., Vatta, P., Ruaro, M.E., Del Sal, G., Parton, R.G., and Schneider, C. 2000. The growth suppressing gas 1 product is a GPI-linked protein. FEBS Lett. 481: 152-158.

Stecca, B., Mas, C., Clement, V., Zbinden, M., Correa, R., Piguet, V., Beermann, F., and Ruiz, I.A.A. 2007. Melanomas require HEDGEHOG-GLI signaling regulated by interactions between GLIl and the RAS-MEK/AKT pathways. Proc. Natl. Acad. Sci. 104: 5895-5900.

Steeg, P.S. 2003. Metastasis suppressors alter the signal transduction of cancer cells. Nat. Rev. Cancer 3: 55-63.

Steeg, P.S. 2006. Tumor metastasis: Mechanistic insights and clinical challenges. Nat. Med. 12: 895-904.

Takaoka, A., Adachi, M., Okuda, H., Sato, S., Yawata, A., Hinoda, Y., Takayama, S., Reed, J.C., and Imai, K. 1997. Anticell death activity promotes pulmonary metastasis of melanoma cells. Oncogene 14: 2971-2977.

Tsai, Y.C., Mendoza, A., Mariano, J.M., Zhou, M., Kostova, Z., Chen, B., Veenstra, T., Hewitt, S.M., Helman, L.J., Khanna, C., et al. 2007. The ubiquitin ligase gp78 promotes sarcoma metastasis by targeting KAIl for degradation. Nat. Med. 13: 1504-1509.

Wetmore, C. 2003. Sonic hedgehog in normal and neoplastic proliferation: Insight gained from human tumors and animal models. Curr. Opin. Genet. Dev. 13: 34-42.

Zamorano, A., Lamas, M., Vergara, P., Naranjo, J.R., and Segovia, J. 2003. Transcriptionally mediated gene targeting of gas1 to glioma cells elicits growth arrest and apoptosis. $I$. Neurosci. Res. 71: 256-263.

Zamorano, A., Mellstrom, B., Vergara, P., Naranjo, J.R., and Segovia, J. 2004. Glial-specific retrovirally mediated gas1 gene expression induces glioma cell apoptosis and inhibits tumor growth in vivo. Neurobiol. Dis. 15: 483-491. 


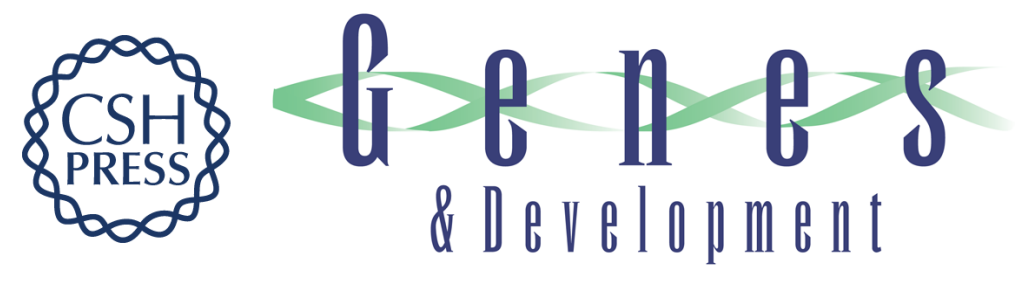

\section{A genome-wide shRNA screen identifies GAS1 as a novel melanoma metastasis suppressor gene}

Stephane Gobeil, Xiaochun Zhu, Charles J. Doillon, et al.

Genes Dev. 2008, 22:

Access the most recent version at doi:10.1101/gad.1714608

Supplemental http://genesdev.cshlp.org/content/suppl/2008/11/05/22.21.2932.DC1
Material

References This article cites 38 articles, 13 of which can be accessed free at:

http://genesdev.cshlp.org/content/22/21/2932.full.html\#ref-list-1

License

Email Alerting

Receive free email alerts when new articles cite this article - sign up in the box at the top

Service

right corner of the article or click here.

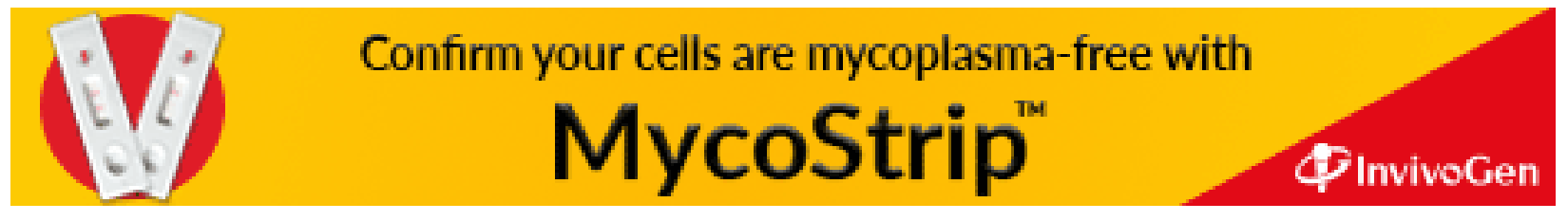

